\title{
Modelling of contaminant migration in unsaturated soils
}

\author{
G. Siracusa, A. D. La Rosa, L. Musumeci \& G. Maiolino \\ Department of Physical and Chemical Methodology for Engineering, \\ Catania University, Italy
}

\begin{abstract}
Laboratory studies are useful for understanding the behaviour of chemical contaminants in soil, although such investigations do not always relate directly to field conditions. This paper reports an application of a model in order to investigate the migration of nitrates through the soil. The model calibration was carried out using the experimental data obtained from two different soil samples collected in the countryside of Catania (Italy). The device used for laboratory scale study was a permeameter loaded with the soil samples; acqueous solutions containing different percentage of nitrate were used as chemical contaminant. Two software products, SEEP/W and CTRAN/W, are used in tandem to analyze the contaminant transport for unsaturated conditions: SEEP/W computes the water flow velocity, volumetric water content, and water flux; CTRAN/W uses these parameters to compute the contaminant migration. Unsaturated zone models are useful tools in predicting the effects of measures and can be used to optimise agricultural practice aiming to minimise the impact on the environment. Keywords: contaminant migration, soil protection, unsaturated zone model, permeability, nitrate concentration.
\end{abstract}

\section{Introduction}

Nitrate is the most abundant anion reported in contaminated groundwater, soils, and sediment due to agricultural practice. A considerable mass of nitrate may accumulate by natural processes in the unsaturated zone (UZ) in arid and semiarid climates, where infiltration of water at the surface is low [1]. Groundwater quality maybe significantly impacted when this sink of nitrate is 
mobilized by activities that enhance natural infiltration to the UZ such as intensive fertilizer applications in agriculture as well as wastewater disposal in infiltration ponds. In general, the unsaturated zone models are useful tools in predicting short or long-term effects of applied measures and can be used to optimise agricultural practice while minimising the impact on the environment [2-5]. Current unsaturated zone models have a varied degree of abstraction concerning the simulation of complex physical and biochemical soil processes $[6,7]$ and for that reason it is not easy to choose a suitable model to estimate the impact of agriculture on groundwater pollution. The choice of using two softwares, CTRAN/W used in conjunction with SEEP/W, makes it possible to analyze problems varying from simple particle tracking in response to the movement of water, to complex processes involving diffusion, dispersion, adsorption, radioactive decay and density dependencies.

\section{Experimental}

\subsection{General description of SEEP/W and CTRAN/W models}

$\mathrm{SEEP} / \mathrm{W}$ is a finite element software product for analyzing groundwater seepage and excess pore-water pressure dissipation problems within porous materials such as soil and rock. SEEP/W can model, in addition to traditional steady-state saturated flow, both saturated and unsaturated flow, that makes it possible to analyze seepage as a function of time and to consider such processes as the infiltration of precipitation. CTRAN/W is a finite element software product that can be used to model the movement of contaminants through porous materials such as soil and rock. CTRAN/W utilizes the SEEP/W flow velocities to compute the movement of dissolved constituents in the pore-water [8].

The inclusion of unsaturated flow in groundwater modelling is important for obtaining physically realistic analysis results. In soils, the hydraulic conductivity and the water content, or water stored, changes as a function of pore-water pressure. SEEP/W models these relationships as continuous functions. Furthermore, many contaminant transport problems may be simplified by using steady-state groundwater flow. In other cases, transient groundwater flow is required. SEEP/W can be used to generate a steady state or transient groundwater flow solution for CTRAN/W.

\subsubsection{Analysis types}

- Particle tracking analysis

Gives an idea of the contaminant travel distances and travel times. While particle tracking is a quick way of presenting the contaminated region, a complete advection dispersion analysis is required to know the concentration within the contaminated region.

- Advection-dispersion analysis.

Advection refers to the process by which solutes are transported by the bulk motion of flowing groundwater. Dispersion refers to the phenomenon of contaminant spreading from the path that it would be expected to follow 
according to the advective hydraulics of the flow system. Virtually all contaminant transport analyses require computation of advection and dispersion.

The differential equation which describes advective-dispersive contaminant transport is known as the advection-dispersion equation. The derivation of the equation should be carried by applying the principle of mass balance to an element of porous medium and considers the processes of advection and dispersion. Additional terms which include the effect of adsorption and decay have also been derived. The following is the one-dimensional form of the advection-dispersion equation (Geo-Slope User's Guide)

$$
\Theta D \frac{\partial^{2} C}{\partial x^{2}}-U \frac{\partial C}{\partial x}-\lambda \Theta C-\lambda S \rho_{d}=\left(\Theta+\rho_{d} \frac{\partial S}{\partial C}\right) \frac{\partial C}{\partial t}
$$

where:

$C=$ concentration

$\Theta=$ volumetric water content

$D=$ hydrodynamic dispersion coefficient

$U=$ Darcian velocity

$S=$ adsorption

$\rho_{d}=$ bulk (dry) mass density of the porous medium

$t=$ time

$x=$ distance in the $x$ direction

The first term in the equation represents transport by dispersion, the second represents transport by advection, the third represents decayed mass loss in the fluid phase, and the fourth represents decayed mass loss in the solid phase. The term on the right side of the equation represents storage of mass in the fluid phase and in the solid phase due to a change in concentration. CTRAN/W implements the two dimensional form of the advection-dispersion equation shown above.

\subsection{Soil samples collection and characterisation}

Two soil samples were collected in two different areas nearby the countryside of Catania: sample 1 is arable land; sample 2 is sandy land. Samples were collected at $30 \mathrm{~cm}$ depth by means of a device fitted with steel tubes $(15 \mathrm{~cm}$ diameter and $60 \mathrm{~cm}$ height).

Soil characterization was carried by determining the following parameters:

- Natural water content

- Texture

- $\quad$ Soil bulk density (mass per unit volume of dry soil in $\mathrm{g} / \mathrm{cm}^{3}$ ).

- Void ratio

- Permeability

Results of soil characterisation are summarized in table 1 . 
Table 1: $\quad$ Results of soil characterisation.

\begin{tabular}{|c|c|}
\hline Sample & Properties \\
\hline $\begin{array}{l}\text { Sample } 1 \\
(10 \% \text { sand; } 16 \% \text { silt; } \\
74 \% \text { clay })\end{array}$ & $\begin{array}{lc}\text { Water content \% } & W=16.20 \\
\text { Specific gravity of soil grains } & G S=2.46 \\
\text { Void ratio } & \begin{array}{l}e=1.096 \\
\text { Porosity }\end{array}=52 \% \\
\text { Permeability } & \mathrm{K}=1.34 \times 10^{-6} \mathrm{~cm} / \mathrm{s} \\
\end{array}$ \\
\hline $\begin{array}{l}\text { Sample } 2 \\
(56 \% \text { sand; } 17 \% \text { silt; } \\
27 \% \text { clay })\end{array}$ & $\begin{array}{lc}\text { Water content \% } & W=33.20 \\
\text { Specific gravity of soil grains } & G S=2.63 \\
\text { Void ratio } & e=0.502 \\
\text { Porosity } & n=33 \% \\
\text { Permeability } & \mathrm{K}=5.2 \times 10^{-6} \mathrm{~cm} / \mathrm{s}\end{array}$ \\
\hline
\end{tabular}

\subsubsection{Tests description}

- The Natural water content

Each samples $(1 \mathrm{~kg})$ was dried in oven at $100^{\circ} \mathrm{C}$ for 24 hours and weighed. The difference between wet soil weight and dried soil weight, multiplied by 100 gives the natural water content of each samples.

- Texture analysis

Soil texture describes the size distribution of individual soil particles. Specifically, texture is defined as the relative distribution of various sized particles. However, texture is generally used to reference the proportions of sand, silt, and clay. The sand was graded by passing through a series of sieves, large to small. The silt and clay fractions are too fine to be separated by sieving; they were separated on the basis of their rate of fall in a liquid. According to the textural triangle, samples were classified as clay soil (sample 1) and sandy-clay loam (sample 2).

- Permeability

Is a measure of the rate at which water can flow through the soil and is expressed in $\mathrm{cm} / \mathrm{sec}$. Soil permeability depends on several factors: the size of soil grains, the properties of pore fluids, the void ratio of the soil, the shapes and arrangement of pores, the degree of saturation. The laboratory method adopted is the variable-head (falling-head) test. This test is used to determine the drainage characteristics of relatively finegrained soils and is generally performed on undisturbed samples [9]. In this test, water is forced, by a falling head pressure, through a soil specimen of known dimensions and the rate of flow is determined. The percolation test was carried out by using $100 \mathrm{ml}$ of a potassium nitrate solution (0.2 molar).

- $\quad$ Void ratio $E=V V / V S$

volume of voids in $\mathrm{cm}^{3}$,

$\mathrm{VV}$ volume of solids in $\mathrm{cm}^{3}, V S$

- $\quad$ Porosity $N=V V / V T$

volume of voids in $\mathrm{cm}^{3}, V V$

total volume in $\mathrm{cm}^{3}, V T$ 


\section{Model implementation}

In order to model the contaminant migration in unsaturated soil, SEEP/W was firstly run. The flow is proportional to the hydraulic gradient and the hydraulic conductivity (coefficient of permeability). After set the geometry (a $10 \mathrm{~m}$ in length $\times 5 \mathrm{~m}$ depth soil) and the grid (132 mesh $1 \times 0.5 \mathrm{~m})$, input data are required: coefficient of permeability, water content, texture (as reported in table 1).

Boundary conditions were specified as total head $(\mathrm{H})$. The SEEP/W CONTOUR function allows one to graphically view the results by displaying velocity vectors that represent the flow direction. A vector is drawn in each element, with the end point of the vector at the centre point of the element. The vector represents the average velocity within the element. The seepage flow velocities computed from SEEP/W are then used by CTRAN/W for the contaminant transport analysis:

- In particle tracking analysis, the dissolved solutes are represented by particles. Figure 1 (a)-(c) is an example of a particle tracking analysis. For each time step, the particles are moved in space proportionally to the water flow velocity and the time step size. The particle flow paths provide a graphical representation of the contaminant plume movement caused by purely advective transport; the effects of dispersion, adsorption, decay and density are not considered. It is possible to view the travel time, location, distance travelled and average speed of a theoretical particle at any point along its flow path.

- In the advection-dispersion analysis, adsorption of contaminant on the soil particles is linearly related to concentration. This is the concept of chemical partitioning between the fluid and solid phases, quantified by a chemical partitioning coefficient. CTRAN/W allows a more general relation to be used to specify the chemical partitioning by allowing the adsorption to be specified as a function of concentration. In effect, this means that the chemical partitioning coefficient, (which is the slope of the adsorption/concentration function), can be specified as a function of concentration. Figure 3 (a)-(d) and Figure 4 (a), (b) are graphical representations of advection-dispersion analysis of a solution of nitrate (starting concentration 100 units/l) flowing respectively through sample 1 and sample 2 .

\section{Results}

Figures 3 and 4 show the simulations of the contaminant migration through two different soil samples (1 and 2). The migration is reported as temporal variation of the contaminant concentration due to water percolation through the soil. The red color indicates the maximum concentration while the blue indicates the minimum concentration. The comparison of simulation results for sample 1 
and 2 shows that the migration of contaminant in the sandy soil is higher, according to the concentration value of 8.5 unit/l, after 48 hours (figure $4(\mathrm{~b})$ ) while the contaminant simulation in the clay soil (sample 1) reports a concentration value of 6 unit/1, after 48 hours (figure 3(d)).

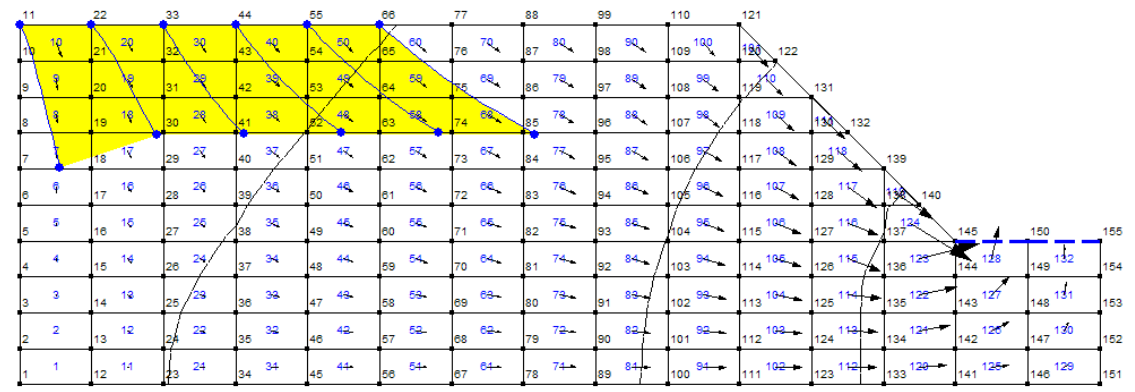

(a)

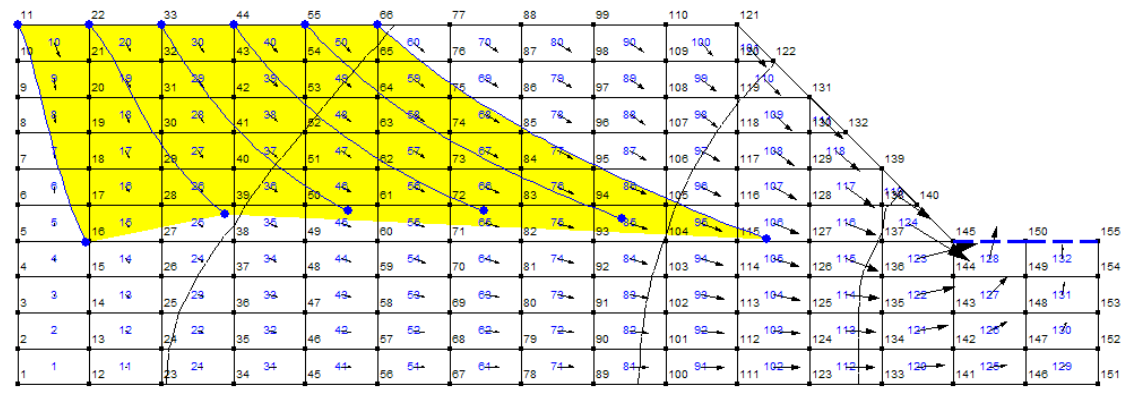

(b)

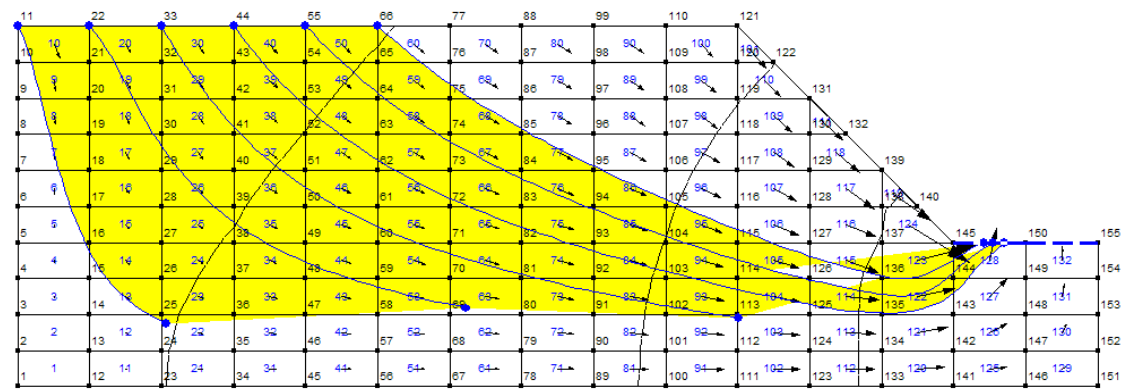

(c)

Figure 1: Particle tracking analysis for sample1: (a) Particle tracking analysis after 12 hours; (b) Particle tracking analysis after 24 hours; (c) Particle tracking analysis after 48 hours. 


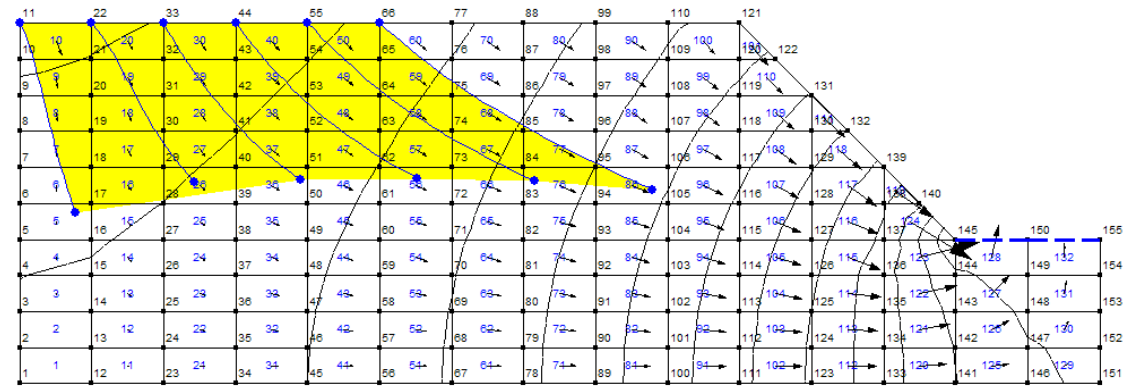

(a)

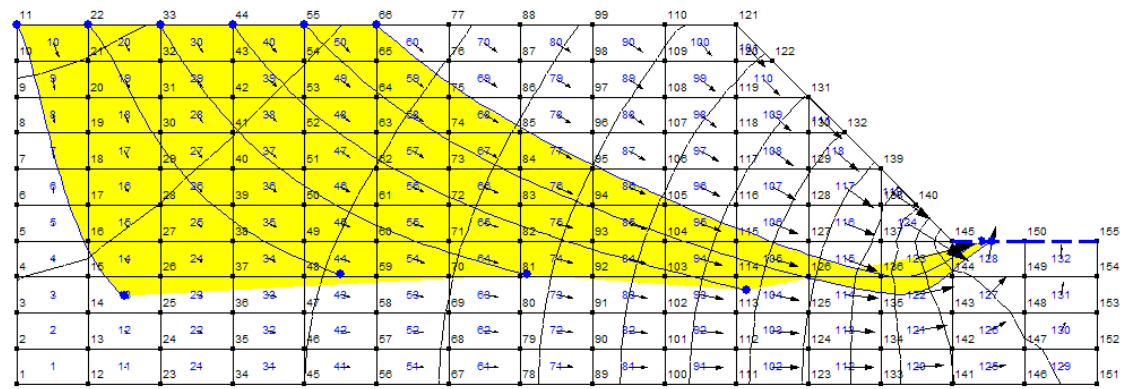

(b)

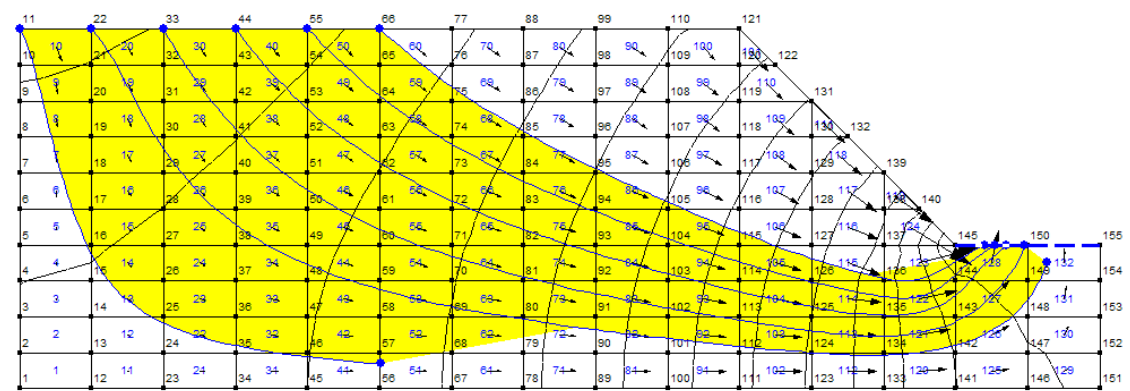

(c)

Figure 2: Particle tracking analysis for sample 2: (a) Particle tracking analysis after 12 hours; (b) Particle tracking analysis after 24 hours; (c) Particle tracking analysis after 48 hours. 

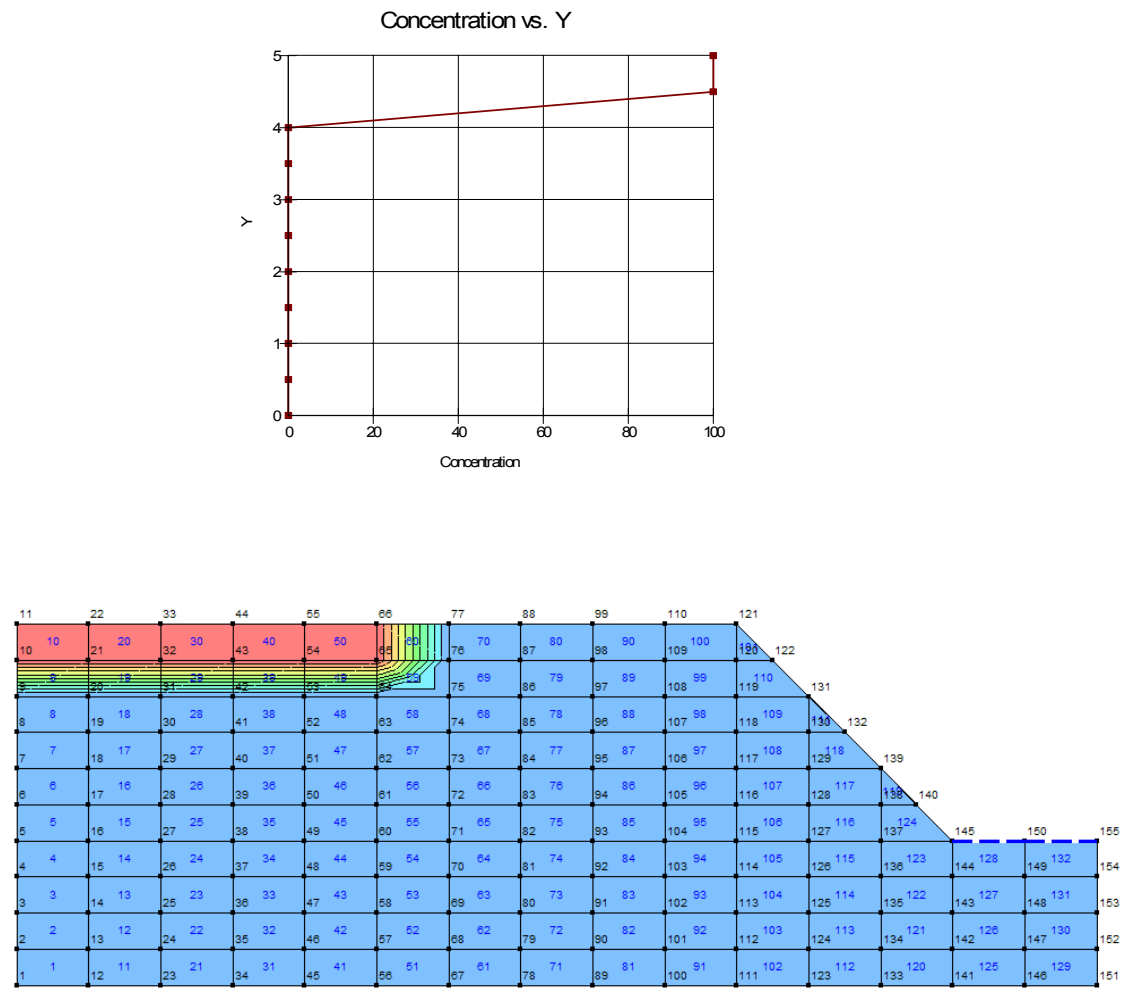

(a)

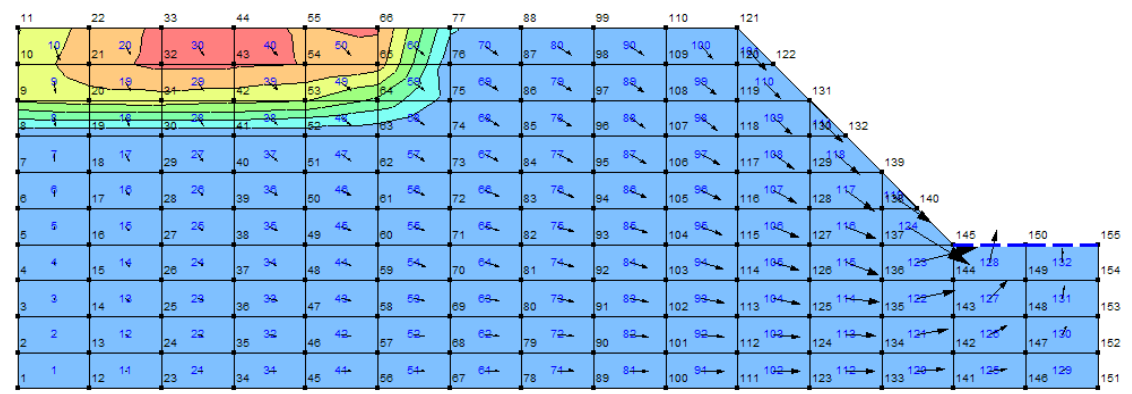

(b)

Figure 3: Advection-dispersion analysis of sample 1; (a) Advectiondispersion analysis, starting; (b) Advection-dispersion analysis after 12 hours; (c) Advection-dispersion analysis, after 24 hours; (d) Advection-dispersion analysis, after 48 hours. 


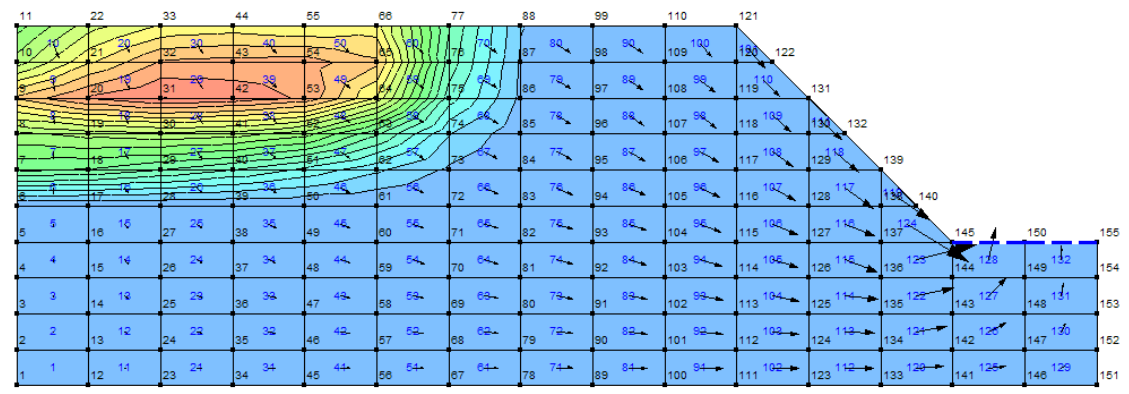

(c)

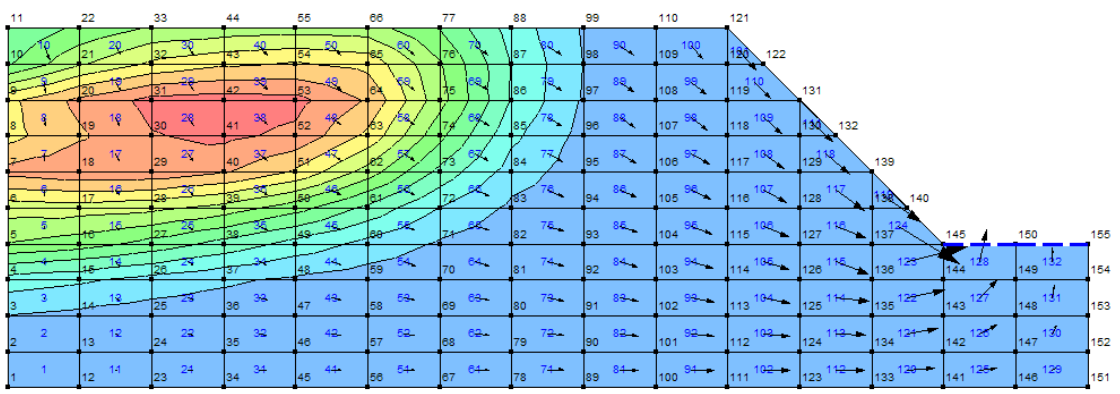

Concentration vs. $Y$

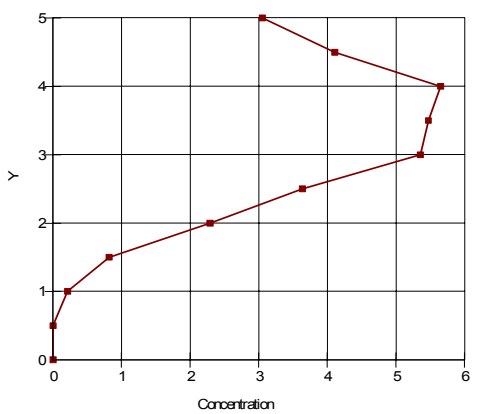

(d)

Figure 3: Continued. 
464 Sustainable Development and Planning III

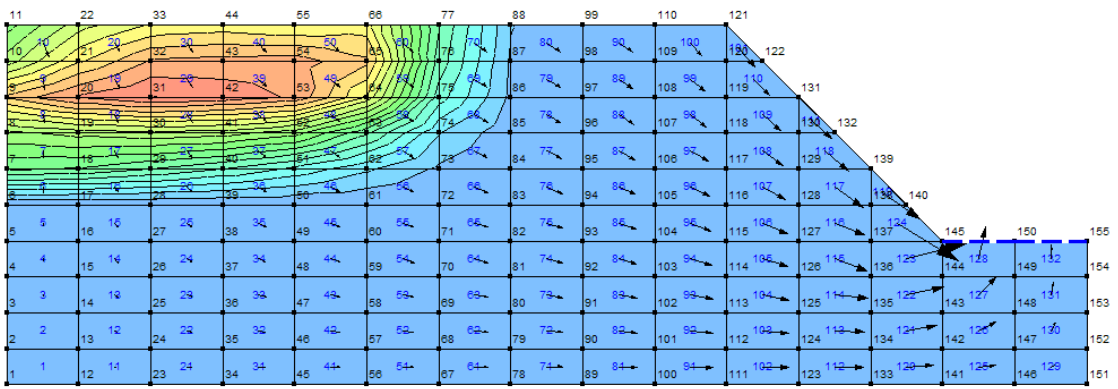

(a)

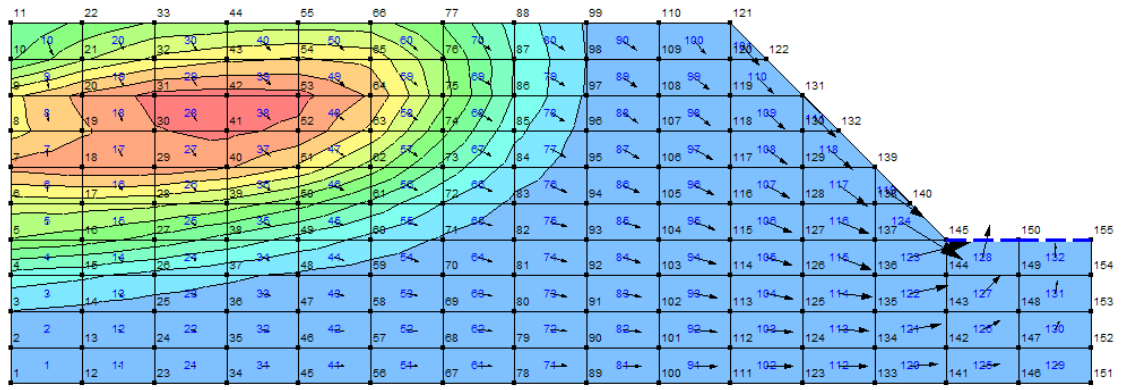

Concentration vs. Y

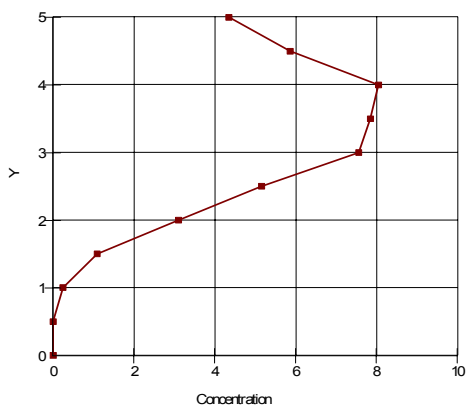

(b)

Figure 4: Advection-dispersion analysis of sample 2; (a) Advectiondispersion analysis, after 24 hours; (b) Advection-dispersion analysis, after 48 hours. 


\section{References}

[1] Walvoord, M. A., Phillips, F. M., Stonestrom, D. A., Evans, R. D., Hartsough, P. C., Newman, B. D., Striegl, R. G., A reservoir of nitrate beneath desert soils, Science, 302, pp.1021-1024, 2003.

[2] Feddes, R.A., Kabat, P., van Bakel, P.J.T., Bronswijk, J.J.B., Halbertsma, J., Modelling soil water dynamics in the unsaturated zone-state of the art. J. Hydrol. 100, pp .69-111, 1988.

[3] Wu, L., McGechan, M.B., A review of carbon and nitrogen processes in four soil nitrogen dynamics models, J. Agric. Eng. Res. 69, pp. 279-305, 1998.

[4] Van Dam, J.C., Feddes, R.A., Numerical simulation of infiltration, evaporation and shallow groundwater levels with Richards equation, $J$. Hydrol. 233, pp. 72- 85, 2000.

[5] Wu, Y.Sh., Forsyth, P.A.,. On the selection of primary variables in numerical formulation for modelling multiphase flow in porous media, $J$. Contam. Hydrol., 48, pp. 277- 304, 2001.

[6] Willigen, de P., Nitrogen turnover in the soil-crop system; comparison of fourteen simulation models. Fertil. Res., 27, pp. 141- 149, 1991.

[7] Diekkruger, B., Sondgerath, D., Kersebaum, K.C., McVoy, C.W., Validity of agroecosystem models. A comparison of results of different models applied to the same data set. Ecol. Model. 83, 3-29, 1995.

[8] GEO-SLOPE User's Guide, CTRAN/W and SEEP/W: http://www.geoslope.com.

[9] California Test 220, State of California, Business, Transportation and Housing Agency, http://www.dot.ca.gov/hq/esc/ctms/CT_220.pdf. 